\title{
TYPE STRUCTURE COMPLEXITY AND DECIDABILITY ${ }^{1}$ \\ BY
}

T. S. MILLAR

\begin{abstract}
We prove that for every countable homogeneous model $\mathscr{Q}$ such that the set of recursive types of $\mathrm{Th}(\mathbb{Q})$ is $\Sigma_{2}^{0}, \mathbb{Q}$ is decidable iff the set of types realized in $\mathbb{Q}$ is a $\Sigma_{2}^{0}$ set of recursive types. As a corollary to a lemma, we show that if a complete theory $T$ has a recursively saturated model that is decidable in the degree of $T$, then $T$ has a prime model.
\end{abstract}

In this paper all models mentioned will be assumed countable. If $\mathbb{Q}$ is homogeneous and realizes either no nonprincipal types [1] or all recursive types [2] then

$\mathbb{Q}$ is decidable iff the set of types realized by $\mathscr{Q}$ is an r.e. set of recursive types.

An examination of the proofs involved leads naturally to the conjecture that the techniques can be combined to prove $(*)$ for those $\mathcal{Q}$ that are simply homogeneous. Unfortunately this is false in general [5]. However, if the structure of recursive types of the theory of $\mathscr{Q}$ is not pathological, then (*) can be proved for those $\mathscr{Q}$ which are simply homogeneous. Specifically, the principal result of this paper is to prove that if $\mathbb{Q}$ is homogeneous and the set of recursive types of the theory of $\mathscr{Q}$ is $\Sigma_{2}^{0}$, then $\mathscr{Q}$ is decidable iff the set of types realized by $\mathscr{Q}$ is a $\Sigma_{2}^{0}$ set of recursive types. Since every complete theory's set of recursive types is $\Pi_{2}^{0}$, the result is the best possible, in light of [5].

Notations and conventions. All types in this paper are assumed complete. A specific effective first order language $L$ is assumed fixed, as well as an effective enumeration $\left\{\sigma_{i} \mid i<\omega\right\}$ of all formulas of the language. An $n$-type $\Gamma$ is recursive if the set $\left\{i \mid \sigma_{i} \in \Gamma\left(x_{0}, \ldots, x_{n-1}\right)\right\}$ is recursive. $\left\{\mu_{i} \mid i<\omega\right\}$ is an effective enumeration of all partial recursive functions $\mu: \omega \rightarrow 2$. An index $e$ for a recursive type $\Gamma$ is a natural number such that $\mu_{e}$ is the characteristic function for $\Gamma$ relative to $\left\{\sigma_{i} \mid i<\omega\right\}$. A set of recursive types is $\Sigma_{1}^{0}\left(\Sigma_{2}^{0}\right)$ if there is a $\Sigma_{1}^{0}\left(\Sigma_{2}^{0}\right)$ set of indices for the types in that set. We will say $\left\{\Gamma_{i} \mid i<\omega\right\}$ is an effective enumeration of types if there is some recursive $f$ such that $f(i)$ is an index for $\Gamma_{i}, i<\omega . \Gamma^{s}$ will denote the first $s$ formulas (order determined by index) of $\Gamma \cap\left\{\sigma_{i} \mid i<\omega\right\} . \theta^{k}=\theta$ if $k=0$, and $\neg \theta$ if $k=1$. $\left\{c_{i} \mid i<\omega\right\}$ will be distinct constant symbols not in $L$ and $\left\{\psi_{i} \mid i<\omega\right\}$ an effective enumeration of all sentences in $L \cup\left\{c_{i} \mid i<\omega\right\}$ such that each sentence occurs

Received by the editors March 14, 1980

1980 Mathematics Subject Classification. Primary 03F50; Secondary 03H13.

${ }^{1}$ The preparation of this paper was partially supported by Grant NSF-MCS-7900824. 
infinitely often. $\left\{\bar{c}_{i} \mid i<\omega\right\}$ will be an effective enumeration of $\left\{c_{i} \mid i<\omega\right\}^{<\omega}$. If $\theta\left(c_{0}, \ldots, c_{n}\right) \in L \cup\left\{c_{i} \mid i<\omega\right\}$ such that all $c_{j}$ 's occurring in $\theta$ are among $\left\{c_{i} \mid i \leqslant n\right\}$, then

$$
\begin{aligned}
\theta^{*}\left[\left(c_{i_{0}}, \ldots, c_{i_{s}}\right)\right]={ }_{\mathrm{df}} & \exists x_{0} \cdots \exists x_{i_{0}-1} \exists x_{i_{0}+1} \cdots \exists x_{i_{s}-1} \exists x_{i_{s}+1} \\
& \ldots \exists x_{n} \theta\left(x_{0}, \ldots, x_{i_{0}-1}, c_{i_{0}}, x_{i_{0}+1}, \ldots, x_{i_{s}-1}, c_{i_{s}}, x_{i_{s}+1}, \ldots, x_{n}\right) .
\end{aligned}
$$

$v: \omega \times \omega \rightarrow \omega$ is a recursive function that is $1-1$ onto and such that if $v(i, j)=n$ then $i \leqslant n .(r)_{i}$ is the exponent of the $i$ th prime in the prime factorization of $r$. Finally, if $F$ is a function with domain $\omega$, then ' $F$ ' ' denotes ' $\operatorname{Lim}_{n \rightarrow \infty} F(n)$ '. For other definitions and conventions, see [2-4].

We begin with several lemmas and interesting corollaries.

LeMma 1. If the set of recursive types of a complete theory is $\Sigma_{2}^{0}$, then it is also $\Sigma_{1}^{0}$.

Proof. This is immediate from Lemma 3 of [2].

By the familiar technique of padding, if $B$ is a set of recursive types with an r.e. set of indices, then it is easy to see that it has a recursive set $A$ of indices. However, to then say that $B$ is recursive would be misleading, since the question of whether or not $n$ is an index of a type in $B$ is not equivalent to whether or not $n$ is in $A$. However, the use of the term r.e. is not similarly misleading.

Lemma 2. Assume that the set of all recursive types of a complete theory $T$ is $\Sigma_{2}^{0}$. Then for every $\Sigma_{2}^{0}$ set $A$ of types of $T$ there is a $\Sigma_{1}^{0}$ set of types $B \supset A$ such that every type in $B-A$ is principal.

Proof. Assume for notational simplicity that $A$ is a set of 1-types. Let $\left\{\varphi_{i}(x) \mid i<\right.$ $\omega\}$ be an effective enumeration of all formulas of $L(T)$ in the one free variable displayed, and let $\left\{\Sigma_{i} \mid i<\omega\right\}$ be an effective enumeration (by Lemma 1) of all recursive 1-types of $T$. Since $A$ is $\Sigma_{2}^{0}$, fix a recursive $R(x, y, n)$ such that $\{n \mid$ $\exists x \forall y R(x, y, n)\}$ is a set of indices for the types in $A$. We will define $f(n, m)$, inductively on $m$ and uniformly in $n$, such that for the recursive $h$ produced by the $s-m-n$ theorem satisfying $f(n, m)=\mu_{h(n)}(m)$ for all $m, n,<\omega$, the range of $h$ will be the r.e. set of indices (relative to $\left\{\varphi_{i} \mid i<\omega\right\}$ ), for the desired $B$. So fix $n<\omega$. We specify that $n$ is active for $m=0$. Assume that $f\left(n, m^{\prime}\right)$ has been defined for $m^{\prime}<m$. If $n$ is active for $m$ then define $f$ according to:

(a) $f(n, m)=\mu_{(n)_{1}}(m)$ if

$$
\exists s \geqslant m\left[\mu_{(n) 1}^{s}(m) \downarrow \text { and } \forall r \leqslant s R\left((n)_{2}, r_{1}(n)_{1}\right)\right]
$$

and

$$
T \vdash \exists x\left[\underset{i<m}{\wedge} \varphi_{i}^{f(n, i)}(x) \wedge \varphi_{m}^{\mu_{(n) 1}(m)}\right] ;
$$

(b) $f(n, m)=0$ if not (a) and

$$
T \vdash \exists x\left[\wedge_{i<m} \varphi_{i}^{f(n, i)}(x) \wedge \varphi_{m}(x)\right] ;
$$

(c) $f(n, m)=1$ otherwise. 
If $n$ is not active at $m$ then:

(i) $f(n, m)=k$ if

$$
T \vdash\left[{ }_{i<m}^{\wedge} \varphi_{i}^{f(n, i)}(x) \rightarrow \varphi_{m}(x)^{k}\right], \quad k=0,1 ;
$$

(ii) $f(n, m)=1-k$ otherwise, where for the least $j$ such that

$$
\bigwedge_{i<m} \varphi_{i}^{f(n, i)} \in \Sigma_{j}, \quad \varphi_{m}^{k} \in \Sigma_{j}, \quad k=0,1 .
$$

If the defining condition is (a) then $n$ is active at $m+1$, otherwise $n$ is inactive.

First we claim that $f$ is recursive. The only condition that is not immediate is when $n$ is active at $m$, since there is an unbounded quantifier in the defining formula of (a). However, if $\mu_{(n)}(m) \uparrow$, then certainly $(n)_{1}$ is not the index of a type. Therefore $\exists r\urcorner R\left((n)_{2}, r,(n)_{1}\right)$, and so the search in (a) would terminate. Next we claim that every type in $A$ has an index in the range of $h$. For suppose $\exists x \forall y$ $R(x, y, v)$. Then for an $r$ such that $\forall y R(r, y, v)$ it is easy to see that $h\left(3^{r} \cdot 2^{v}\right)$ is an index for the type in $A$ with index $v$. Finally, assume that a nonprincipal recursive type $\Sigma_{j} \notin A$ has an index $h(n)$, in order to obtain a contradiction. Fix the least subscripted such $\Sigma_{j_{0}}$. Since $\Sigma_{j_{0}} \notin A$, fix a $y$ such that $\neg R\left((n)_{2}, y,(n)_{1}\right)$. So by condition (a), $n$ is not active for $m>y$. By the choice of $j_{0}$ there is an $s_{0}>y$ such that $\wedge_{i<s_{0}} \varphi_{i}{ }^{f(n, i)} \notin \Sigma_{j}, j<j_{0}$. Since $\Sigma_{j_{0}}$ is nonprincipal, there is a $\varphi_{p}, p>s_{0}$, such that

$$
T \forall\left[\hat{i<s}_{i<s_{i}} \varphi^{f(n, i)}(x) \rightarrow \varphi_{p}^{k}\right], \quad k=0,1 .
$$

Then for some $m \leqslant p$ we must have $\bigwedge_{i \leqslant m} \varphi_{i}^{f(n, i)} \notin \Sigma_{j_{0}}$, the desired contradiction. This completes the lemma.

COROLlaRY 1. If the set of recursive types of a complete decidable theory $T$ is $\Sigma_{2}^{0}$, then $T$ has a prime model.

Proof. First we take $A$ to be empty in the previous lemma. However, we modify the subsequent proof so that for $\varphi_{n}$ consistent with $T, f(n, j)$ is defined so that

$$
T \vdash \exists x\left[\wedge_{i<n} \varphi_{i}^{f(n, i)}(x) \wedge \varphi_{n}(x)\right], \quad f(n, n)=0 .
$$

Then $f(n, m)$ is defined as in the construction for $m>n$. Thus $h(n)$ will be the index of a principal type containing $\varphi_{n}$, for those $\varphi_{n}$ consistent with $T$. By well-known results this implies that $T$ has a prime model. In fact, by [1] the prime model of $T$ is then decidable. Note that this corollary is applicable to arbitrary complete theories, except of course everything must be relativized to the degree of the theory involved.

COROLlaRY 2. If a complete theory has a recursively saturated model which is decidable in the degree of the theory, then the theory has a prime model.

Proof. Every type of a complete theory $T$ which is recursive in the degree of $T$ is realized in every recursively saturated model of $T$. Also, if a model $\mathscr{Q}$ is decidable in 
some degree a, then the set of types realized in $\mathbb{Q}$ is r.e. in the degree a. Now just apply the relativized version of the previous corollary. Again by [1], the prime model is actually decidable in the degree of the theory.

THEOREM. Assume that the set of recursive types of $\mathrm{Th}(\mathbb{Q})$ is $\Sigma_{2}^{0}$ and $\mathbb{Q}$ is homogeneous. Then $\mathbb{Q}$ is decidable iff the set of types realized in $\mathbb{Q}$ is a $\Sigma_{2}^{0}$ set of recursive types.

Proof. The 'only if' is immediate. For the other direction fix, by Lemmas 1 and 2, effective enumerations $\left\{\Sigma_{i} \mid i<\omega\right\}$ and $\left\{\Gamma_{i} \mid i<\omega\right\}$ of all recursive types of $\operatorname{Th}(\mathscr{Q})$ and of those types realized in $\mathcal{Q}$, respectively. It is sufficient to construct a decidable homogeneous model $\mathcal{C}$ realizing exactly the set of types $\left\{\Gamma_{i} \mid i<\omega\right\}$. In fact, we will only construct the complete diagram of such a model. This will be done by a Henkin construction that a stage $t$ inductively determines a $\theta_{t} \in\left\{\psi_{i} \mid i<\omega\right\}$. The complete diagram will be $\left\{\theta_{i} \mid i<\omega\right\}$. We adopt the abbreviation $\chi_{j}=_{\mathrm{df}} \wedge_{i<j} \theta_{i}$. Partial recursive functions $f_{i}, g_{i, j}: \omega \rightarrow\left\{\bar{c}_{i} \mid i<\omega\right\}, H_{i}: \omega \rightarrow\left\{\Gamma_{i} \mid i<\omega\right\}$ will also be inductively defined during the course of the construction. In terms of these functions we define the partial recursive $A_{i}: \omega \rightarrow\left\{\bar{c}_{j} \mid j<\omega\right\}, i<\omega$ by $A_{0}(s)=\langle\rangle$, and $A_{5 t+i}(s)$ is to be the smallest indexed $\bar{c}_{i}$ such that

$$
\begin{aligned}
& A_{5 t+1}(s)=A_{5 t}(s) \hat{f} f_{t}(s) \\
& A_{5 t+2}(s)=A_{5 t+1}(s)\left\langle c_{t}\right\rangle ; \\
& A_{5 t+3}(s)=A_{5 t+2}(s) \\
& A_{5 t+4}(s)=A_{5 t+3}(s) g_{i, j}(s), \text { where } v(i, j)=t ; \text { and } \\
& A_{5 t+5}(s)=A_{5 t+4}(s) ;
\end{aligned}
$$

and of course if there can be no such $\bar{c}_{i}$, then $A_{5 t+1}(s)$ is undefined.

Choices during the construction will be influenced by a set of requirements $\left\{R_{i} \mid i<\omega\right\}$ having the natural priority ordering. Loosely speaking, the task associated with requirement $R_{5 n+i}$ will be, for

$i=0$ : to ensure that $\Gamma_{n}$ is realized in $\mathcal{C}$;

$i=1,4$ : to ensure that candidates associated with requirements of higher priority have their respective types amalgamated by a $\Gamma_{j}$;

$i=2$ : to ensure that $\left\langle c_{0}, \ldots, c_{n}\right\rangle$ realizes a $\Gamma_{j}$; and

$i=3$ : to ensure that $C$ is homogeneous.

Specifically, we say that $R_{5 n+i}$ is $t$-satisfied for $\varphi$ if

$$
\begin{aligned}
& i=0: \Gamma_{n}\left(f_{n}(t)\right) \cup\left\{\chi_{t}, \varphi\right\} \text { is consistent; } \\
& i=1: H_{3 n}(t)\left(A_{5 n+1}(t)\right) \cup \Gamma_{n}^{t}\left(f_{n}(t)\right) \cup H_{3 n-1}(t)^{t}\left(A_{5 n}(t)\right) \cup\left\{\chi_{t}, \varphi\right\} \text { is consistent; }
\end{aligned}
$$$$
i=2: H_{3 n+1}(t)\left(A_{5 n+2}(t)\right) \cup H_{3 n}(t)^{t}\left(A_{5 n+1}(t)\right) \cup\left\{\chi_{t}, \varphi\right\} \text { is consistent; }
$$

$i=3$ : If $v(k, j)=n$ and $\Gamma_{j}\left(\left\langle c_{0}, \ldots, c_{k}\right\rangle \bar{x}\right) \cup\left\{\chi_{t}, \varphi\right\}$ is consistent, where $\Gamma_{j}$ is a $k+v$-type for some $v>0$, then $\Gamma_{j}\left(\left\langle c_{0}, \ldots, c_{k}\right\rangle g_{k, j}(t)\right) \cup\left\{\chi_{t}, \varphi\right\}$ is consistent; or if either of the previous conditions fail, i.e. $\Gamma_{j}\left(\left\langle c_{0}, \ldots, c_{k}\right\rangle \bar{x}\right)$ is inconsistent or $\Gamma_{j}$ is an $m$-type for some $m \leqslant k$, then $g_{k, j}(t)=\langle\rangle$;

$i=4$ : if $v(k, j)=n$ and $g_{k, j}(t) \neq\langle\rangle$, then

$$
H_{3 n+2}(t)\left(A_{5 n+4}(t)\right) \cup H_{3 n+1}(t)^{t}\left(A_{5 n+2}(t)\right) \cup \Gamma_{j}^{t}\left(\left\langle c_{0}, \ldots, c_{k}\right\rangle g_{k, j}(t)\right) \cup\left\{\chi_{t}, \varphi\right\}
$$

is consistent; and if the condition fails, then the definition automatically holds. 
Notice that to determine whether or not $R_{m}$ is $t$-satisfied for $\psi_{i}$ is a procedure uniformly effective in $m, t$, and $i$, as long as the construction is uniformly effective. Various requirements and functions will be associated in an obvious way: $R_{5 n}-f_{n}$; $R_{5 n+1}-f_{n}, H_{3 n}$, and $A_{5 n+1} ; R_{5 n+2}-H_{3 n+1}, A_{5 n+2}$, and $A_{5 n+3} ; R_{5 n+3}-g_{i, j}$, where $v(i, j)=n$; and $R_{5 n+4}-H_{3 n+2}, A_{5 n+4}$, and $A_{5 n+5}$. In the construction that follows, if a function is defined on an argument $t$ and has not previously been specified as undefined for $t+1$, then its value on $t+1$ is to be the same as its value on $t$. Also, requirements are either active or inactive at a particular stage.

\section{The construction.}

Stage 0 . All functions are undefined at 0 (except $A_{0}$ ), all requirements are inactive, and $\theta_{0}={ }_{\mathrm{df}}\left(c_{0}=c_{0}\right)$.

Stage $t=3 s+1$. If $\theta_{t} \chi_{t}=\exists x \sigma(x)$, then for the least indexed $c_{i}$ not occurring in $\theta_{t}={ }_{\mathrm{df}} \sigma\left(c_{i}\right)$; otherwise $\theta_{t}={ }_{\mathrm{df}}\left(c_{0}=c_{0}\right)$.

Stage $t=3 s+2$. Fix the highest priority requirement $R_{5 n+i}, i<5$, that is inactive. This requirement is now active and

$i=0$. If $\Gamma_{n}$ is a $k$-type, then define $f_{n}(t)$ to be the least indexed $\bar{c}_{m}$ that is a $k$-tuple and no $c_{j}$ occurring in $\bar{c}_{m}$ occurs in $\chi_{t}$ or $\psi_{s}$.

$i=1$. If $A_{3 n+i}(3 s)$ is a $k$-tuple, then let $H_{3 n}(t)$ be the least indexed $\Gamma_{m}$ that is a $k$-type and such that

$$
\Gamma_{m}\left(A_{3 n+1}(3 s)\right) \cup \Gamma_{n}^{3 s}\left(f_{n}(3 s)\right) \cup H_{3 n-1}(3 s)^{3 s}\left(A_{3 n}(3 s)\right) \cup\left\{\chi_{t}\right\}
$$

is consistent.

$i=2$. If $H_{3 n}(3 s)$ is a $k$-type, then let $H_{3 n+1}(t)$ be the least indexed $\Gamma_{m}$ that is a $k+1$-type and such that

$$
\Gamma_{m}\left(A_{5 n+2}(3 s)\right) \cup H_{3 n}(3 s)^{3 s}\left(A_{5 n+1}(3 s)\right) \cup\left\{\chi_{t}\right\}
$$

is consistent.

$$
i=3 \text {. Let } v^{-1}(n)=\langle k, j\rangle \text {. If } \Gamma_{j} \text { is an } r \text {-type } r \leqslant k+1 \text { or } \Gamma_{j}\left(\left\langle c_{0}, \ldots, c_{k}\right\rangle \bar{x}\right) \cup\left\{\chi_{t}\right\}
$$
is inconsistent, then $g_{k, j}(t)=_{\mathrm{df}}\langle\rangle$. Otherwise define $g_{k, j}(t)$ as the least indexed $\bar{c}_{m}$ that is a $(p-k-1)$-tuple, where $\Gamma_{j}$ is a $p$-tuple, and such that no $c_{u}$ occurring in $\bar{c}_{m}$ occurs in $\chi_{t}$ or $\psi_{3 s}$.

$i=4$. If $v^{-1}(n)=\langle k, j\rangle$ and $g_{k, j}(3 s)=\langle\rangle$, then $H_{3 n+2}(t)=H_{3 n+2}(3 s+2)$; otherwise, if $A_{5 n+4}(3 s)$ is an $r$-tuple, then let $H_{3 n+2}(t)$ be the least indexed $\Gamma_{m}$ that is an $r$-type and such that

$$
\Gamma_{m}\left(A_{5 n+4}(3 s)\right) \cup H_{3 n+1}(3 s)^{3 s}\left(a_{5 n+2}(3 s)\right) \cup \Gamma_{j}^{3 s}\left(\left\langle c_{0}, \ldots, c_{k}\right\rangle g_{k, j}(3 s)\right) \cup\left\{\chi_{t}\right\}
$$

is consistent.

Regardless of the value of $i, \theta_{t}={ }_{\mathrm{df}}\left(c_{0}=c_{0}\right)$.

Stage $t=3 s+3$. I. There is an active $R_{5 n+2}$ such that $R_{i}$ is $t$-satisfied for $\psi_{s}^{k}$, $k=0,1, i<5 n+2 ; R_{5 m+1}$ is not $t$-satisfied for $7\left(\left(\chi_{t} \wedge \psi_{s}^{k}\right)^{*}\left[A_{5 m+1}(3 s)\right]\right)$ for either $k=0$ or $k=1$ (neither value of $k$ produces $t$-satisfaction), $m<n$; and $R_{5 n+1}$ is $t$-satisfied for $\urcorner\left(\left(\chi_{t} \wedge \psi_{s}^{k}\right)^{*}\left[A_{5 n+2}(3 s)\right]\right)$ for at least one of $k=0,1$. Fix the greatest such $n$. If $A_{5 n+2}(3 s)$ is an $r$-tuple, then let $\Sigma_{m}$ be the least indexed $\Sigma_{j}$ that is an $r$-type and such that $\Sigma_{m}\left(A_{5 n+2}(3 s)\right) \cup\left\{\chi_{t}\right\}$ is consistent. [ $R_{5 n+2}$ will be referred to as the controlling requirement.] 
A. $\Sigma_{m}\left(A_{5 n+2}(3 s)\right) \cup\left\{\chi_{t}, \psi_{s}^{k}\right\}$ is inconsistent for some $k=0,1$ (actually at most one). For the least such $k, \theta_{t}={ }_{\mathrm{df}} \psi_{s}^{k}$.

B. Otherwise. Then $\theta_{t}={ }_{\mathrm{df}}\left(c_{0}=c_{0}\right)$.

II. Otherwise. Let $m_{k}$ be the least $i$ such that $R_{i}$ is not $t$-satisfied for $\psi_{s}^{k}$, or $m_{k}=t$ if no such $i \leqslant t$ exists, $k=0,1$. Now let $k=0$ if $m_{0} \geqslant m_{1}, k=1$ otherwise, and $\theta_{t}={ }_{\mathrm{df}} \psi_{s}^{k}$.

After $\theta_{t}$ has been defined let $R_{5 m+i}$ be the requirement of highest priority that is not $(t+1)$-satisfied for $\left(c_{0}=c_{0}\right)$. Then $R_{j}$ is now inactive and all associated functions are undefined for $j \geqslant 5 m+i$, and if $i=1$ or $i=4$, then also for $j=5 m+i-1$.

This ends the construction.

LEMMA 3. The construction is uniformly effective.

Proof. The details of this will be left to the reader, but we note that all types considered are (uniformly) recursive and that if $\varphi(\bar{x}) \in L$ is a formula consistent with $\operatorname{Th}(\mathscr{Q})$, then there is always an $i<\omega$ such that $\varphi(\bar{x}) \in \Gamma_{i}(\bar{x})$.

LEMMA 4. $\left\{\theta_{i} \mid i<\omega\right\} \cup \operatorname{Th}(\mathbb{Q})$ is consistent.

Proof. This is also easy to check, since in fact $\theta_{t}$ is always specified so that $\Gamma_{0}\left(A_{1}^{*}\right) \cup\left\{\chi_{t+1}\right\}$ is consistent.

Lemma 5. $f_{i}^{*}, g_{i, j}^{*}, H_{i}^{*}, A_{i}^{*}$ all exist and $\left\{\theta_{i} \mid i<\omega\right\}_{\uparrow} L \cup \operatorname{rg} A_{i}^{*}$ is complete, $i, j<\omega$.

Proof. The proof is by induction on the index of the associated $R_{i}$. Simultaneously we will prove that for all $n<\omega$ there is a $t<\omega$ such that for all

$$
\varphi \in\left\{\psi_{i} \mid i<\omega\right\}_{\mid L \cup \operatorname{rg} A_{5 n+2}^{*}} H_{3 n}^{*}\left(A_{5 n+1}^{*}\right)+\left[\chi_{t} \rightarrow \varphi^{k}\right] \text { for some } k=0,1 .
$$

Since $R_{0}$ has the highest priority it is easy to see from the construction that $f_{0}^{*}=f_{0}(1)$ and $H_{0}^{*}=1$. Now, for each $\psi_{j} \in L \cup \operatorname{rg} f_{0}^{*} R_{0}$ is $(t+1)$-satisfied for $\psi_{j}^{k}$ for exactly one value of $k=0,1$. Since $R_{0}$ has the highest priority, it follows from the instructions at stages $3 s+3$ that for that value of $k, \psi_{j}^{k} \in\left\{\theta_{i} \mid i<\omega\right\}$. This proves the lemma for $R_{0}$ and $R_{1}$. So assume that the lemma is true for $R_{i}, i<5 n$. Thus $H_{3 n-1}^{*}, A_{3 n}^{*}$ exist and $\left\{\theta_{i} \mid i<\omega\right\}_{\mid L \cup \mathrm{rg} A_{3 n}^{*}}$ is complete. Thus from the construction it is obvious that $\left\{\theta_{i} \mid i<\omega\right\}_{\uparrow} \cup \cup \mathrm{rg} A_{3 n}^{*}$ is in fact $H_{3 n-1}^{*}\left(A_{3 n}^{*}\right)$. Since $H_{3 n-1}^{*}$ and $\Gamma_{n}$ are both types that are realized in $\mathcal{C}$, there is a $\Gamma_{k}$ such that $H_{3 n-1}^{*}(\bar{x}) \cup \Gamma_{n}(\bar{y}) \subset$ $\Gamma_{k}(\bar{x}, \bar{y})$. (By the choice made in the case $i=0$ of stages $3 s+2$ for $f_{n}(3 s+2)$, and the construction, it is not necessary to invoke homogeneity in order to justify what follows.) Fix the least such $k=k_{1}$. Let $t_{0}$ be a stage after which every $R_{i}$ is always active, $i<5 n$. Let $t_{1}>t_{0}$ be a stage such that for each $i<k_{1}, H_{3 n-1}^{* t_{1}}(\bar{x}) \cup \Gamma_{n}^{t_{1}}(\bar{y}) \phi$ $\Gamma_{i}(\bar{x}, \bar{y})$. Then it is easy to see by the above remark, the construction, and the choice of $t_{0}, t_{1}$ that for all $t>t_{1}+2 H_{3 n}^{*}=\Gamma_{k_{1}}, f_{n}(t)=f_{n}(t+1)$ and $A_{5 n+1}(t)=$ $A_{5 n+1}(t+1)$. This completes $R_{5 n}$ and $R_{5 n+1}$ except to show that $\left\{\theta_{i} \mid i<\omega\right\}_{\uparrow} L \cup \operatorname{rg} A_{5 n+1}^{*}$ is complete. By the induction hypotheses fix $t_{2}>t_{1}$ such that for all $m<n$

for all $\varphi \in\left\{\psi_{i} \mid i<\omega\right\}_{\mid L \cup \operatorname{rg} A_{5 m+2}^{*}}$ there is a $k=0,1$ such that $H_{3 m}^{*}\left(A_{5 m+1}^{*}\right)+\left[\chi_{t_{2}} \rightarrow \varphi^{k}\right]$. 
Next fix $\psi_{s} \in L \cup \operatorname{rg} A_{5 n+1}^{*}$ in order to show that $\psi_{s}^{k} \in\left\{\theta_{i} \mid i<\omega\right\}$ for some $k=0,1$. By the assumption on the enumeration $\left\{\psi_{i} \mid i<\omega\right\}$, we may assume without loss that $s>t_{2}$. Now consider stage $3 s+3$. It is enough to show that the defining case is II, since then $\theta_{3 s+3}$ is $\psi_{s}^{k}$ for one of $k=0,1$. Suppose first that for some $m<n R_{5 m+2}$ is the controlling condition in case I. Then for some value of $k=0,1$ \urcorner$\left(\left(\chi_{3 s+3} \psi_{s}^{k}\right)^{*}\left[A_{5 m+2}^{*}\right]\right)$ does $(3 s+3)$-satisfy $R_{5 m+1}$. Thus by the choice of $t_{2}$ and (*) it follows that $H_{5 m}^{*}\left(A_{5 m+1}^{*}\right) \vdash \chi_{t_{2}} \rightarrow \neg\left(\left(\chi_{3 s+2} \wedge \psi_{s}^{k}\right)^{*}\left[A_{5 m+2}^{*}\right]\right)$ for that value of $k$. But then it is easy to see that $R_{5 m+1}$ is not $(3 s+3)$-satisfied for $\psi_{s}^{k}$, which contradicts the instructions in I. On the other hand $\psi_{s}^{k}$ is in $L \cup \operatorname{rg} A_{5 n+1}^{*}$ and so $n o R_{5 m+2}$ for $m>n$ can be the controlling condition either, since $R_{5 n+1}$ can only be $(3 s+3)$ satisfied for $\psi_{s}^{k}$ for at most one value of $k$. Thus the defining case is II.

In order to prove the lemma for $R_{5 n+2}$, note first that $A_{5 n+2}^{*}=A_{5 n+1}^{*} \hat{\langle}\left\langle c_{n}\right\rangle$; so fix $t_{3}>t_{2}$ such that for all $t \geqslant t_{3} A_{5 n+1}(t)=A_{5 n+1}^{*}$. The completeness of $\left\{\theta_{i} \mid i<\omega\right\}_{1 L \cup \mathrm{rg} A_{5 n+2}^{*}}$ is the first claim to be established. Therefore choose any $\psi_{s} \in L \cup \operatorname{rg} A_{5 n+2}^{*}$ in order to show that $\psi_{s}^{k} \in\left\{\theta_{i} \mid i<\omega\right\}$ for one of $k=0,1$. Again we may assume that $s>t_{3}$ and just as in the last paragraph the only difficulty possible is that the defining condition at stage $3 s+3$ is I.B. However, the argument is identical to the one in the previous paragraph for eliminating the possibility that $R_{5 m+2}$ is the controlling requirement for $m \neq n$. Thus the only new alternative is that $R_{5 n+2}$ might be the controlling requirement. But then since $\psi_{s} \in L \cup \operatorname{rg} A_{5 n+2}^{*}$, $\psi_{s}^{k}$ can be consistent with the appropriate $\Sigma_{m}\left(A_{5 n+2}^{*}\right)$ in I. for at most one value of $k$. Thus the defining condition would be I.A. and so the claim is proven.

We next establish the additional inductive assumption that was asserted, i.e. that (*) holds for $m=n$. Assume that it fails in order to obtain a contradiction; thus for every $t<\omega$ there is a $\psi_{s} \in L \cup \operatorname{rg} A_{5 n+2}^{*}$ such that

$$
H_{3 n}^{*}\left(A_{5 n+1}^{*}\right) \forall\left(\chi_{t} \rightarrow \psi_{s}^{k}\right), \quad k=0,1 .
$$

Now by Lemma 1 and what has just been established, $\left\{\theta_{i} \mid i<\omega\right\}_{1 L \cup \mathrm{rg} A_{5 n+2}^{*}}$ is a recursive type of $\operatorname{Th}(\mathbb{Q})$ and so is a $\Sigma_{i}$ for some $i<\omega$. Let $m$ be the least such $i$. By the choice of $m$ fix $t_{4}>t_{3}$ such that either $\Sigma_{i}$ is not a $k$-type, where $A_{5 n+2}^{*}$ is a $k$-tuple, or $\Sigma_{i}\left(A_{5 n+2}^{*}\right) \cup\left\{\chi_{t_{4}}\right\}$ is inconsistent, $i<m$. By our assumption, there is a $\psi_{s} \in L \cup \operatorname{rg} A_{5 n+2}^{*}$ satisfying $H_{3 n}^{*}\left(A_{5 n+1}^{*}\right) \forall\left(\chi_{t_{4}} \rightarrow \psi_{s}^{k}\right), k=0,1$. Fix the least indexed such $\psi_{s}$ satisfying $3 s+3>t_{4}$. Assume first that there is a least $j, t_{4} \leqslant j<3 s$ +3 such that $H_{3 n}^{*}\left(A_{5 n+1}^{*}\right)+\left[\left(\chi_{j} \wedge \theta_{j}\right) \rightarrow \psi_{s}^{k}\right]$ for some $k=0,1$. Thus $H_{3 n}^{*}\left(A_{5 n+1}^{*}\right) \forall$ $\left[\chi_{j} \rightarrow \psi_{s}^{k}\right], k=0,1$. From the construction it follows that $j=3 r+3$ for some $r<s$ and that the defining condition at stage $j$ is not I.B. Since $\psi_{s} \in L \cup \operatorname{rg} A_{5 n+2}^{*}$, it also follows by the choice of $j$ that

$$
H_{3 n}^{*}\left(A_{5 n+1}^{*}\right) \forall\left[\chi_{j} \rightarrow\left(\chi_{j} \wedge \theta_{j}\right) *\left[A_{5 n+2}^{*}\right]\right] .
$$

Thus $R_{5 n+1}$ is $t$-satisfied for $\neg\left(\left(\chi_{j} \wedge \theta_{j}\right)^{*}\left[A_{5 n+2}^{*}\right]\right)$. In fact, the claim is now that $R_{5 n+1}$ is the controlling condition for stage $j$. To see this note that by $(\dagger)$ and the induction hypothesis (*) that $R_{5 u+1}$ is $j$-satisfied for $\psi_{s}^{k}$ and is not $j$-satisfied for

$$
\neg\left(\left(x_{j} \wedge \psi_{s}^{k}\right) *\left[A_{5 u+2}^{*}\right]\right), \quad k=0,1, u<n .
$$

By the instructions in I of the construction it follows that $R_{5 n+2}$ is the controlling requirement. However, since in the case considered we are not in defining condition 
I.B., it follows that $\theta_{i}$ is specified so that $\chi_{j+1}$ is inconsistent with $\Sigma_{m}\left(A_{5 n+2}^{*}\right)$ (since $\left.j \geqslant t_{4}\right)$. This contradicts the choice of $\Sigma_{m}$. So we are reduced to the case where $H_{3 n}^{*}\left(A_{5 n+1}^{*}\right) \forall\left(\chi_{3 s+3} \rightarrow \psi_{s}^{k}\right), k=0,1$. But again the same argument as before shows that the controlling requirement at stage $3 s+3$ would then be $R_{5 n+2}$. Of course the guarantee that the defining condition is not I.B. is not the same, but there still is one. This is simply because $\psi_{s} \in L \cup \operatorname{rg} A_{5 n+2}^{*}$ and so $\psi_{s}^{k}$ can be consistent with $\Sigma_{m}\left(A_{5 n+2}^{*}\right)$ for at most one value of $k$. Therefore $\theta_{3 s+3}$ is determined so that $\chi_{3 s+4}$ is inconsistent with $\Sigma_{m}\left(A_{5 n+2}^{*}\right)$, which is again a contradiction. This establishes (*) for $m=n$.

So let $t_{4}>t_{3}$ be large enough that (*) holds at stage $t_{4}$. Now there must be some $\Gamma_{i}$ such that $H_{3 n}^{*}\left(A_{5 n+1}^{*}\right) \cup \Gamma_{i}\left(A_{5 n+2}^{*}\right) \cup\left\{\chi_{t_{4}+1}\right\}$ is consistent and $\Gamma_{i}$ is a $k$-type (as before). Fix the least such $i$ to be $r$. Then it is easy to see that $H_{3 n+1}^{*}=\Gamma_{r}$, since $R_{5 s+2}$ can never be the controlling requirement for $s \leqslant n$ after stage $t_{4}$.

So now fix $t_{5}>t_{4}$ such that for all $t>t_{5}, H_{3 n+1}^{*}=H_{3 n+1}(t)$ as we move on to $R_{5 n+3}$ and $R_{5 n+4}$. Let $v^{-1}(n)=\left\langle k_{0}, j_{0}\right\rangle$. The choice of $v$ ensures that

$$
\left\{\theta_{i} \mid i<\omega\right\}_{i L \cup\left\{c, i \leqslant k_{0}\right\}} \subset\left\{\theta_{i} \mid i<\omega\right\}_{i L \cup \operatorname{rg} A_{n_{n+2}^{*}}^{*}}
$$

and is thus a complete type $\Gamma_{i_{0}}$ for some $i_{0}$. If $\Gamma_{i_{0}}\left(c_{0}, \ldots, c_{k_{0}}\right) \not \subset \Gamma_{j_{0}}\left(\left\langle c_{0}, \ldots, c_{k_{0}}\right\rangle \wedge \bar{x}\right)$, then it is easy to see that $g_{k_{0}, j_{0}}^{*}=\langle\rangle$ and $H_{3 n+2}^{*}=H_{3 n+1}^{*}$. If it is a subset, then since $Q$ is homogeneous, there is an $r$ such that

$$
\Gamma_{j_{0}}\left(\left\langle c_{0}, \ldots, c_{k_{0}}\right\rangle \wedge \bar{x}\right) \cup H_{3 n+1}^{*}\left(A_{5 n+2}^{*}\right) \subset \Gamma_{r}\left(A_{5 n+2}^{*} \wedge \bar{x}\right) .
$$

For the least such $r$ there is at least $t_{6}>t_{5}$ such $H_{3 n+2}^{*}=\Gamma_{r}$ and $A_{5 n+4}^{*}=A_{5 n+4}\left(t_{6}\right)$. These claims as well as the completeness of $\left\{\theta_{i} \mid i<\omega\right\}_{i} L \cup_{\mathrm{rg}} A_{3_{n+4}}^{*}$ are similar to previous ones in their proof and we leave them to the reader. This completes the lemma.

LeMma 6. $\left\{\theta_{i} \mid i<\omega\right\}$ is complete and recursive.

Proof. Lemmas 3 and 5.

Lemma 7. $\left\{\boldsymbol{\theta}_{i} \mid i<\omega\right\}$ is the complete diagram of a decidable model $\mathrm{C}$.

Proof. Lemmas 4 and 6 and the Henkin construction.

Lemma 8. Every $\Gamma_{i}$ is realized in $\mathcal{e}, i<\omega$.

Proof. This follows from Lemma 5 since in fact $\Gamma_{i}$ is realized in $C$ by the equivalence class of $f_{i}^{*}$.

Lemma 9. Only the types in $\left\{\Gamma_{i} \mid i<\omega\right\}$ are realized in $\mathcal{C}$.

Proof. This again follows from Lemma 5 since by that lemma $H_{i}^{*}$ exists for all $i<\omega$. In particular, $H_{3 n+1}^{*}$ exists for all $n<\omega$.

LeMma 10. $\mathcal{C}$ is homogeneous.

Proof. Suppose $\Gamma_{r}(\bar{x}) \subset \Gamma_{j}(\bar{x}, \bar{y})$ and $\left\langle c_{i_{0}}, \ldots, c_{i_{r}}\right\rangle$ realizes $\Gamma_{r}$ in $\mathcal{C}$. Then fix a $j_{0}$ such that $\Gamma_{j}\left(\left\langle x_{i_{0}}, x_{i_{1}}, \ldots, x_{i_{r}}\right\rangle \wedge \bar{y}\right) \subset \Gamma_{j_{0}}\left(\left\langle x_{0}, x_{1}, \ldots, x_{i_{r}}\right\rangle \wedge \bar{y}\right)$ and $\left\langle c_{0}, \ldots, c_{i_{r}}\right\rangle$ realizes $\Gamma_{j_{0}}(\bar{x}, \bar{y})_{i, \bar{x}}$. By Lemma $5 g_{i_{r}, j_{0}}^{*}$ exists, and by the construction it follows that 
$\left\langle c_{0}, \ldots, c_{i_{r}}\right\rangle \hat{g}_{i_{r}, j_{0}}^{*}$ realizes $\Gamma_{j_{0}}$ in $\mathcal{C}$, and thus $\left\langle c_{i_{0}}, \ldots, c_{i_{r}}\right\rangle g_{i_{r}, j_{0}}^{*}$ realizes $\Gamma_{j}$ in $\mathcal{C}$. This completes the proof of the theorem.

COROllary 3. Assume that $T$ has a decidable recursively saturated model. Then for every homogeneous model $Q$ of $T, Q$ is decidable iff the set of type realized by $Q$ is a $\Sigma_{2}^{0}$ set of recursive types.

Proof. The recursively saturated decidable model provides an effective enumeration of all recursive types of $T$.

\section{BIBLIOGRAPHY}

1. L. Harrington, Recursively presentable prime models, J. Symbolic Logic 392 (1974), 305-309.

2. T. S. Millar, Foundations of recursive model theory, Ann. Math. Logic 13 (1978), 45-72.

3. C. C. Chang and H. J. Keisler, Model theory, American Elsevier, New York, 1973.

4. H. Rogers, Jr., Theory of recursive functions and effective compatibility, McGraw-Hill, New York, 1967.

5. T. S. Millar, Homogeneous models and decidability, Pacific J. Math. 91 (1980), 407-418.

Department of Mathematics, University of Wisconsin, Madison, Wisconsin 53706 